\title{
Dietary supplementation of dried plum: a novel strategy to mitigate heat stress in broiler chickens
}

\author{
Sanjeev Wasti, Nirvay Sah, Amit K. Singh, Chin N. Lee, Rajesh Jha and Birendra Mishra* (1)
}

\begin{abstract}
Background: Heat stress is a significant problem in the poultry industry, causing a severe economic loss due to its detrimental effects on chickens' health and performance. Dried plum (DP) is a good source of minerals, vitamins, antioxidants, and phenolic compounds. Studies have suggested that DP has several health benefits, such as maintaining the body's redox system, immune status, and calcium hemostasis. Based on the health benefits of DP, we hypothesized that the dietary supplementation of DP would alleviate the detrimental effects of heat stress on broiler chickens.
\end{abstract}

Results: To test the hypothesis, day-old broiler chicks $(n=72)$ were randomly allocated to three treatment groups ( $n=24 /$ group): no heat stress (NHS), heat stress (HS), and heat stress with dried plum (HS + DP), and reared under standard conditions. The inclusion of 2.5\% DP in the feed of the HS + DP group was made during the treatment period, while birds in other groups were provided with a standard finisher diet. After 21 days, birds in the HS and HS + DP groups were exposed to cyclic heat stress conditions $\left(33^{\circ} \mathrm{C}\right.$ for $8 \mathrm{~h}$ during daytime) for 3 weeks, while those in the NHS group were reared under normal conditions $\left(22-24^{\circ} \mathrm{C}\right)$. Weekly body weight and feed intake were recorded to calculate the average daily gain (ADG), average daily feed intake (ADFI), and feed conversion ratio (FCR). Heat stress significantly decreased the final body weight, ADG, ADFI, and increased FCR compared to the NHS group, whereas dietary supplementation of DP significantly improved these growth performance parameters compared to the HS group. Furthermore, supplementation of DP significantly increased the expression of heat shock protein-related genes (HSF1, HSF3, HSP70, and HSP90), antioxidant-related genes (SOD1, SOD2, GPX1, GPX3, $P R D X 1$, and TXN), tight junction-related genes (CLDN1, and OCLN), and immune-related genes (IL4, MUC2) in the ileum as compared to the HS group. The microbiota analysis showed significant enrichment of Bacillales, Christensenellaceae, Bacillaceae, Peptostreptococcaceae, and Anaerotruncus in heat-stressed birds supplemented with DP as compared to the HS group. Further, DP supplementation also significantly increased the concentration of acetate, propionate, and total VFA in the cecal digesta of the HS + DP group as compared to the HS group.

Conclusion: These findings suggest that DP supplementation effectively improved the growth performances and gut health parameters in the heat-stressed birds. Thus, dried plum can be a potential feed supplement to mitigate heat stress in broiler chickens.

Keywords: Dried plum, Gene expression, Heat stress, Microbiota, Mitigation

\footnotetext{
*Correspondence: bmishra@hawaii.edu

Department of Human Nutrition Food and Animal Sciences, University of Hawaii at Manoa, Honolulu, HI 96822, USA
}

(c) The Author(s). 2021 Open Access This article is licensed under a Creative Commons Attribution 4.0 International License, which permits use, sharing, adaptation, distribution and reproduction in any medium or format, as long as you give appropriate credit to the original author(s) and the source, provide a link to the Creative Commons licence, and indicate if changes were made. The images or other third party material in this article are included in the article's Creative Commons. licence, unless indicated otherwise in a credit line to the material. If material is not included in the article's Creative Commons licence and your intended use is not permitted by statutory regulation or exceeds the permitted use, you will need to obtain permission directly from the copyright holder. To view a copy of this licence, visit http://creativecommons.org/licenses/by/4.0/ The Creative Commons Public Domain Dedication waiver (http://creativecommons.org/publicdomain/zero/1.0/) applies to the data made available in this article, unless otherwise stated in a credit line to the data. 


\section{Introduction}

High environmental temperature is a significant problem in the poultry industry, affecting the health and performance of poultry, which is expected to worsen due to increasing global warming. It is estimated that heat stress results in an annual economic loss of \$128 to \$165 million to the US poultry industry [1]. Heat stress in poultry results in several physiological changes such as oxidative damage, acid-base imbalance, and suppressed immunocompetence, leading to reduced feed intake, poor feed efficiency, and reduced body weight, poor meat quality, increased susceptibility to diseases, and higher mortality [2]. Poultry meat is the most consumed and widely accepted animal protein worldwide, and its demand is ever increasing to meet the growing population. There has been a huge improvement in chicken genetics over the past decades to meet this demand [3]. These improved strains have higher metabolic rates and production performances [4] and are prone to higher environmental temperatures.

It is practically impossible to avoid heat stress in poultry farms; however, several strategies have been successfully applied to improve the deleterious effects of heat stress in poultry [2]. As environmental heat stress exerts its detrimental effects by inducing oxidative stress, various strategies such as supplementation of vitamins, minerals, antioxidants, and plant extracts have been used to reduce oxidative stress in poultry [5]. Phytochemicals with antioxidant activity have been emerged as a better solution to improve heat stress in poultry $[2,6]$.

Dried plum (Prunus domestica L.) has the highest oxygen radical absorbance capacity (ORAC) score among the 22 most commonly consumed vegetables and fruits $[7,8]$. DP also contains a reasonable amount of antioxidants (fat-soluble carotenoids, alpha-tocopherol, etc.), polyphenolic compounds (such as chlorogenic acids, proanthocyanidins, etc.), sorbitol, and fibers [9]. Moreover, DP is a good source of several vitamins (vitamin A, $\mathrm{C}, \mathrm{K}_{1}, \mathrm{~B}_{1}, \mathrm{~B}_{2}$, and niacin) and minerals $(\mathrm{Ca}, \mathrm{K}, \mathrm{Mg}$, Se, and $\mathrm{Zn}$ ) [9] (Table S1). DP, a mixture of such bioactive compounds, is known to minimize oxidative stress in rodents [7]. Besides its antioxidant role, DP has several beneficial effects on gut health, calcium metabolism, and immune function $[9,10]$. Several in vitro studies have highlighted the effectiveness of DP to prevent free radical damage as well as inflammatory responses [11, 12]. DP also serves as a supplemental feed additive due to its wide availability in different parts of the world at a reasonable price, considering the claimed benefits. However, there is no reported study on the effectiveness of DP in ameliorating heat stress effects in poultry. Based on the noted health benefits of DP, we hypothesized that the dietary supplementation of DP would mitigate the detrimental effects of heat stress on gut health and growth performances in poultry. This study aimed to determine the mitigatory effects of DP on growth performance and the gut health parameters in heat-stressed broiler chickens.

\section{Material and methods \\ Animals and husbandry practices}

All the animal experiments were carried out following the approved protocol from the University of Hawaii Institutional Animal Care and Use Committee (IACUC) (Approval No.17-2605). The dose of DP (2.5\%) was chosen based on its antioxidant contents and the dose rate used in the human and rodent studies. Day-old Cob-500 unsexed chicks $(n=72)$ were sourced from a local hatchery, weighed individually, winged tagged, and placed equally and randomly in 18 pens ( 4 birds per pen), making 6 replicates of each treatment $(n=24$ per treatment). Bodyweight (average weight of 40-41 g) was considered to allocate the chicks in each treatment group. The treatment groups were: 1) no heat stress with basal diet (NHS), 2) heat stress with basal diet (HS), and 3) heat stress with basal diet and dried plum (HS + DP) (Fig. S1). Birds were raised on the floor pen system following the standard broiler rearing guidelines for the first 21 d. After $21 \mathrm{~d}$, birds in the HS and HS + DP groups were exposed to $33-35^{\circ} \mathrm{C}$ (during the day- 8:00 to 18:00) and $21-22^{\circ} \mathrm{C}$ (during the night) with $50 \%$ relative humidity for 3 weeks. Birds in the NHS group were reared in identical rooms as $\mathrm{HS}$, and $\mathrm{HS}+\mathrm{DP}$ groups at standard room temperature $\left(22-24^{\circ} \mathrm{C}\right)$ with $50 \%$ relative humidity. Birds were monitored twice daily (in the morning and the evening) for health conditions. A completely randomized design was used in this study. The pen's size was $1 \mathrm{~m} \times 0.61 \mathrm{~m}$, and the stocking density was $1500 \mathrm{~cm}^{2} /$ bird. The lighting regime was $23 \mathrm{~h}$ light and $1 \mathrm{~h}$ dark period.

\section{Diet}

Birds were provided with ad libitum feed and water. The diets were prepared in two phases: starter (1-21 d) and finisher $(22-42 \mathrm{~d})$ to meet the nutrients requirements of broilers [13]. Birds were provided with the standard starter diet for the first $14 \mathrm{~d}$. Afterward, from 14 to $21 \mathrm{~d}$, the inclusion of $2.5 \%$ of DP was made in the starter diet in the HS + DP, while the other two groups were provided with the standard starter diet. From 21 to $42 \mathrm{~d}$, NHS and HS birds were provided with the standard finisher diets, and the inclusion of $2.5 \%$ DP was made in the standard finisher diet of the HS + DP group (Table 1). The available nutrient profile of the DP [9] is presented in Table S1. 
Table 1 Ingredients and nutrient composition of the experimental diets

\begin{tabular}{|c|c|c|c|c|}
\hline \multirow[b]{2}{*}{ Ingredients, \% } & \multicolumn{2}{|l|}{ Starter } & \multicolumn{2}{|l|}{ Finisher } \\
\hline & Standard & With DP & Standard & With DP \\
\hline Corn & 54.86 & 53.36 & 63.14 & 61 \\
\hline SBM & 39.5 & 38.5 & 29.6 & 29 \\
\hline Dried plum & 0 & 2.5 & 0 & 2.5 \\
\hline Soybean oil & 2 & 2 & 4.5 & 4.74 \\
\hline Limestone & 1.27 & 1.27 & 0.85 & 0.85 \\
\hline Monocalcium phosphate & 0.75 & 0.75 & 0.5 & 0.5 \\
\hline Lysine & 0.23 & 0.23 & 0.18 & 0.18 \\
\hline Methionine & 0.14 & 0.14 & 0.12 & 0.12 \\
\hline Threonine & 0.2 & 0.2 & 0.16 & 0.16 \\
\hline $\mathrm{NaCl}$ & 0.43 & 0.43 & 0.35 & 0.35 \\
\hline Sodium bicarbonate & 0.12 & 0.12 & 0.1 & 0.1 \\
\hline Vitamin + Mineral mix* & 0.5 & 0.5 & 0.5 & 0.5 \\
\hline \multicolumn{5}{|c|}{ Calculated nutrient contents, \% } \\
\hline MEn, kcal/kg & 2909 & 2903 & 3203 & 3207 \\
\hline $\mathrm{CP}$ & 22.09 & 21.96 & 18.07 & 18.08 \\
\hline $\mathrm{Ca}$ & 0.75 & 0.75 & 0.52 & 0.52 \\
\hline Total P & 0.57 & 0.56 & 0.47 & 0.46 \\
\hline $\operatorname{dig} P$ & 0.30 & 0.30 & 0.23 & 0.23 \\
\hline Lysine & 1.39 & 1.36 & 1.10 & 1.08 \\
\hline Methionine & 0.48 & 0.47 & 0.41 & 0.41 \\
\hline Cystine & 0.43 & 0.41 & 0.38 & 0.37 \\
\hline Threonine & 1.03 & 1.01 & 0.85 & 0.83 \\
\hline Tryptophan & 0.33 & 0.32 & 0.26 & 0.25 \\
\hline Methionine + Cysteine & 0.91 & 0.89 & 0.8 & 0.78 \\
\hline Arginine & 1.61 & 1.57 & 1.31 & 1.28 \\
\hline Valine & 1.22 & 1.19 & 1.03 & 1.00 \\
\hline Isoleucine & 0.93 & 0.91 & 0.76 & 0.75 \\
\hline Leucine & 1.89 & 1.84 & 1.63 & 1.59 \\
\hline Choline, mg/kg & 1419 & 1382 & 1200 & 1170 \\
\hline dig Lys & 1.25 & 1.22 & 0.99 & 0.97 \\
\hline dig Met & 0.45 & 0.45 & 0.39 & 0.38 \\
\hline dig Thr & 0.85 & 0.83 & 0.69 & 0.68 \\
\hline NDF & 9.13 & 8.89 & 8.78 & 8.53 \\
\hline CF & 3.97 & 4.29 & 3.46 & 3.8 \\
\hline $\mathrm{Na}$ & 0.22 & 0.22 & 0.18 & 0.18 \\
\hline $\mathrm{Cl}$ & 0.30 & 0.30 & 0.25 & 0.25 \\
\hline
\end{tabular}

*Providing the following (per $\mathrm{kg}$ of diet): vitamin $\mathrm{A}$ (trans-retinyl acetate), 10,000 IU; vitamin $D_{3}$ (cholecalciferol), $3000 \mathrm{IU}$; vitamin $\mathrm{E}$ (all-rac-tocopherolacetate), $30 \mathrm{mg}$; vitamin $B_{1}, 2$ mg; vitamin $B_{2}, 8$ mg; vitamin $B_{6}, 4$ mg; vitamin $B_{12}$ (cyanocobalamin), $0.025 \mathrm{mg}$; vitamin $\mathrm{K}_{3}$ (bisulphatemenadione complex), 3 $\mathrm{mg}$; choline (choline chloride), $250 \mathrm{mg}$; nicotinic acid, $60 \mathrm{mg}$; pantothenic acid (D-calcium pantothenate), $15 \mathrm{mg}$; folic acid, $1.5 \mathrm{mg}$; betaíne anhydrous, $80 \mathrm{mg}$; $D$-biotin, $0.15 \mathrm{mg}$; zinc $(\mathrm{ZnO}), 80 \mathrm{mg}$; manganese $(\mathrm{MnO}), 70 \mathrm{mg}$; iron $\left(\mathrm{FeCO}_{3}\right)$, $60 \mathrm{mg}$; copper $\left(\mathrm{CuSO}_{4} \cdot 5 \mathrm{H}_{2} \mathrm{O}\right), 8 \mathrm{mg}$; iodine $(\mathrm{KI}), 2 \mathrm{mg}$; selenium $\left(\mathrm{Na}_{2} \mathrm{SeO}_{3}\right), 0.2 \mathrm{mg}$

\section{Growth performance}

Birds were weighed individually at the start of experiments and were placed in replicate pens. Then, weekly (7, 14, 21, 28, 35, and $42 \mathrm{~d}$ ) body weight and feed intake per replicate pen were recorded. Based on these data, average daily gain (ADG), average daily feed intake (ADFI), and feed conversion ratio (FCR) were calculated.

\section{Sample collection}

At the end of the animal experiment (42 d), two birds from each pen (12 per treatment) were euthanized using carbon dioxide asphyxiation. The sampling was done after a few hours of heat stress during $8 \mathrm{~h}$ high temperature. For the ileal gene expression study, small pieces of the ileum $(5 \mathrm{~cm}$ proximal to the ileocecal junction) were collected ( $n=6$ per treatment; one from each pen), snap-frozen, and stored at $-80{ }^{\circ} \mathrm{C}$ until RNA extraction. For the ileum histomorphology $(n=4$ per treatment), approximately $1 \mathrm{~cm}$ of the ileum sample $(6 \mathrm{~cm}$ proximal to the ileocecal junction) was excised and flushed with $0.9 \%$ normal saline to clear the intestinal digesta. The ileal tissues were sent to the histology core facility at John A. Burns School of Medicine of the University of Hawaii at Manoa for embedding, sectioning, and staining with Hematoxylin and Eosin (H\&E). For the microbiota characterization ( $n=6$ per treatment; one from each pen) and VFA analysis ( $n=12$ per treatment; two from each pen), the cecum was excised and wrapped separately in aluminum foil and was snapped frozen at $-80{ }^{\circ} \mathrm{C}$. Tissues were fixed overnight in $10 \%$ neutral buffered formalin (NBF, pH 7).

\section{Quantitative real-time PCR (qPCR)}

Total RNAs were isolated from the frozen tissues (50$100 \mathrm{mg}$ ) using TRIzol reagent (Invitrogen, Carlsbad, CA, USA) according to the manufacturer's instructions. The total RNA concentration was measured using NanoDrop One (Thermo Fisher Scientific, Madison, WI, USA). The quality of the RNA was determined by running samples on $2 \%$ agarose gel. The RNA samples were stored at $-80^{\circ} \mathrm{C}$ until further analysis. The expressions of candidate genes were analyzed using qPCR as previously described [14]. Specific primer pairs for detecting each gene were designed using the NCBI Primer-Blast tool (Table S2). Briefly, complementary DNA (cDNA) was synthesized from $1 \mu \mathrm{g}$ of total RNA $(20 \mu \mathrm{L}$ reaction of $\mathrm{RT}$ mixture) using a High-Capacity cDNA Reverse Transcription Kit (Applied Biosystems, Foster City, CA, USA) and further diluted with nuclease free-water (1:25). The qPCR was performed using PowerUp SYBR Green Master Mix (Applied Biosystems, Foster City, CA, USA) on a StepOne Plus real-time PCR system (Applied Biosystems). The qPCR reaction mixture consisted of $3 \mu \mathrm{L}$ of cDNA, $5 \mu \mathrm{L}$ PowerUp SYBR Green Master Mix, and $1 \mu \mathrm{L}$ of each 
forward and reverse primers $(5 \mu \mathrm{mol}$ concentration) to make a final reaction mixture of $10 \mu \mathrm{L}$. The qPCR reaction was carried out following standard cycling mode. A melting curve was also generated to confirm SYBR Green-based objective amplicon. Further, each primer pair's specificity was determined by running the qPCR products on $1 \%$ gel electrophoresis. The three different housekeeping genes: glyceraldehyde 3-phosphate dehydrogenase (GAPDH), beta-actin ( $\beta$-actin), and TATA-box binding protein (TBP), were analyzed in triplicate in all treatment groups. $\beta$-actin was the most stable housekeeping genes in the ileum, and it was used for the normalization of gene expression. The target genes were analyzed in duplicates, and an average value was taken for each experimental replicate. The expression levels of candidate genes in this study were determined using the cycle threshold $(\mathrm{Ct})$ values following the standard curve method after normalization with $\beta$-actin. The fold change for each gene was calculated by the $2^{-\Delta \Delta \mathrm{Ct}}$ method.

\section{Ileum histomorphology}

Ileal tissues fixed in 10\% NBF were first dehydrated with a series of ethanol solutions $(70 \%, 80 \%, 95 \%$, and $100 \%)$ and were finally embedded in paraffin as described previously [15]. The embedded ileal tissue was sectioned at $6 \mu \mathrm{m}$ thickness and stained with H\&E. A total of 6 intact, well-oriented villus-crypt units were selected in triplicate (18 measurements for each sample). Sections were observed under an $8 \times$ objective lens, and images were taken using an Olympus microscope (U-TV0.63XC, Tokyo, Japan). Different intestinal morphological parameters such as villus height (VH)- distance from the tip of villus to the crypt, crypt depth (CD)- distance from villus base to the submucosa, and the ratio of villus height to crypt depth $(\mathrm{VH} / \mathrm{CD})$ were measured by using Infinity Analyze software (Lumenera Corporation, Ottawa, ON, Canada). The apparent villus surface area (VSA) was calculated using the formula proposed by [16].

$$
\begin{aligned}
\text { VSA }= & \left(\text { villus width at } 1 / 3^{\text {rd }}+\text { villus width at } 2 / 3^{\text {rd }} \text { of the villus height }\right) \\
& \times 2^{-1} \times \text { villus height }
\end{aligned}
$$

\section{Volatile fatty acids (VFA)}

VFA was analyzed as previously described [17]. Briefly, $200 \mathrm{mg}$ of the cecal content was weighed in the Eppendorf tube. Then, $100 \mu \mathrm{L}$ of an internal standard, Trimethyl acetate (TMA), and $200 \mu \mathrm{L}$ of $25 \%$ metaphosphoric acid were added. Finally, distilled water was added to the tube to make the final volume of $1500 \mu \mathrm{L}$. The tube was then vortexed to homogenize the sample and was centrifuged at $12,000 \times g$ for $15 \mathrm{~min}$ at $4{ }^{\circ} \mathrm{C}$. Afterward, $500 \mu \mathrm{L}$ of the supernatant was transferred in the GC vial and was analyzed using a gas chromatograph (TRACE 1300 Gas Chromatograph; Thermo Scientific, Waltham, MA, USA) coupled with a $30 \mathrm{~m} \times 0.53 \mathrm{~mm}$ internal diameter fused silica capillary column with polar free acid phase (Stabilwax-DA, Restek Corporation, Bellefonte, PA, USA) and a flame ionization detector. The injector-port and flame ionization detector temperatures were fixed at $200^{\circ} \mathrm{C}$ and $240{ }^{\circ} \mathrm{C}$, respectively. In the temperature program, the initial temperature was held at $120^{\circ} \mathrm{C}$ for $4 \mathrm{~min}$ after injection and then increased at $4{ }^{\circ} \mathrm{C} / \mathrm{min}$ to $160^{\circ} \mathrm{C}$, where it was held for 4 min. Helium was used as a carrier gas. The injection volume was set at $0.5 \mu \mathrm{L}$, and analyses were performed. The run time for each analysis was set for $15 \mathrm{~min}$. An aqueous stock standard solution was prepared with different concentrations of $0,0.5,1,2,4,6$, and $8 \mathrm{mmol} / \mathrm{L}$ with a final volume of $1500 \mu \mathrm{L}$. All the standard stock solutions were stored at $-20^{\circ} \mathrm{C}$ until used. Data handling and processing were performed on ChromeleonTM 7.2 software (Thermo Scientific, Waltham, MA, USA).

\section{DNA extraction and 16S rRNA gene sequencing}

Total genomic DNA was extracted from the cecal content using the QIAamp ${ }^{\circ}$ DNA Stool Mini Kit (Qiagen, Hilden, Germany) following the manufacturer's instruction. The concentration and integrity of bacterial DNA were determined by NanoDrop One (Thermo Fisher Scientific, Madison, WI, USA) and agarose gel electrophoresis, respectively. Amplification of the V3-V4 hypervariable regions of the $16 \mathrm{~S}$ rRNA gene was carried out at the University of Hawaii at Manoa Advanced Studies in Genomics, Proteomics, and Bioinformatics core facility as outlined in the Illumina 16S Metagenomic Sequencing Library guideline (Illumina) with the following modification. Platinum Taq DNA Polymerase High Fidelity (Invitrogen, Life Technologies Corporation, Grand Island, NY) was used to set up the PCR reaction, Mag-Bind Total Pure NGS beads (Omega Bio-Tek) were used for PCR Clean-Ups and 35 cycles were used in PCR. Finally, amplicons were normalized, pooled, and sequenced on the Illumina MIseq sequencer.

\section{DNA sequence analysis}

Microbial bioinformatics analysis was carried out by using CLC Genomics Workbench 12.0.1, and the CLC Microbial Genomics module. The sequencing analysis procedures were followed as described in the OTU clustering step by step tutorial (Qiagen, Hilden, Germany). Briefly, the demultiplexed sequences as fastq files were imported in the CLC workbench, which was then paired, trimmed, and filtered to remove lower coverage reads. The filtered reads were then clustered as operational taxonomical units (OTUs), based on 97\% sequence similarity against the Greengenes v13_8 97\% database using 
the CLC Microbial Genomics module. For alpha and beta diversity analysis, the phylogenetic tree was constructed using a maximum-likelihood approach based on multiple sequence alignment (MSA) of the OTUs sequences generated by MUSCLE in the workbench. The alpha diversity was estimated by calculating Simpson's index, and Shanon entropy and was visualized by a boxplot. Beta diversity was estimated by calculating unweighted UniFrac and weighted UniFrac distances and was visualized by principal coordinate analysis (PCoA). Permutational multivariate analysis of variance (PERM ANOVA) procedure was carried out to measure the significance of beta diversity. Differentially abundant taxa (order, family, and genus) were identified using one-way ANOVA on the OTU table after removing OTUs with a lower abundance of less than 10 , and mean separation between the treatment groups was done by using Fisher's Least significant difference (LSD) test in the R-studio.

\section{Statistical analyses}

The growth performance, gene expression, VFA, and ileum histology data were analyzed using the R-studio and are presented as mean \pm SEM. The mean comparisons between different treatment groups were carried out by the Tukey-Kramer test function after performing a one-way analysis of variance (ANOVA). KruskalWallis pairwise test for alpha diversity and PERM ANOVA test for beta diversity were used in the CLC Microbial Genomics module. The Spearman correlation analysis was carried out to determine the association between changed parameters with the differentially enriched microbial species using statistical software JMP v14 (SAS Institute Inc., Cary, NC, USA). Statistical significance was set at $P<0.05$.

\section{Results}

\section{Growth performance}

There was no significant change $(P>0.05)$ in body weight between the treatment groups until $28 \mathrm{~d}$ (Fig. 1). However, from $35 \mathrm{~d}$ onwards, total body weight was significantly decreased $(P<0.05)$ in heat-stressed birds compared to the NHS group. Simultaneously, the supplementation of DP significantly increased the body weight in the heat-stressed birds as compared to the HS group. ADG was significantly decreased $(P<0.05)$ in the heat-stressed birds from $21 \mathrm{~d}$ as compared to the NHS group, and DP significantly improved $(P<0.05)$ the ADG from $28 \mathrm{~d}$ as compared to the HS group. ADFI was significantly lower $(P<0.05)$ in the heat-stressed birds from $21 \mathrm{~d}$ as compared to the NHS, while DP significantly improved $(P<0.05)$ the ADFI from $28 \mathrm{~d}$ in

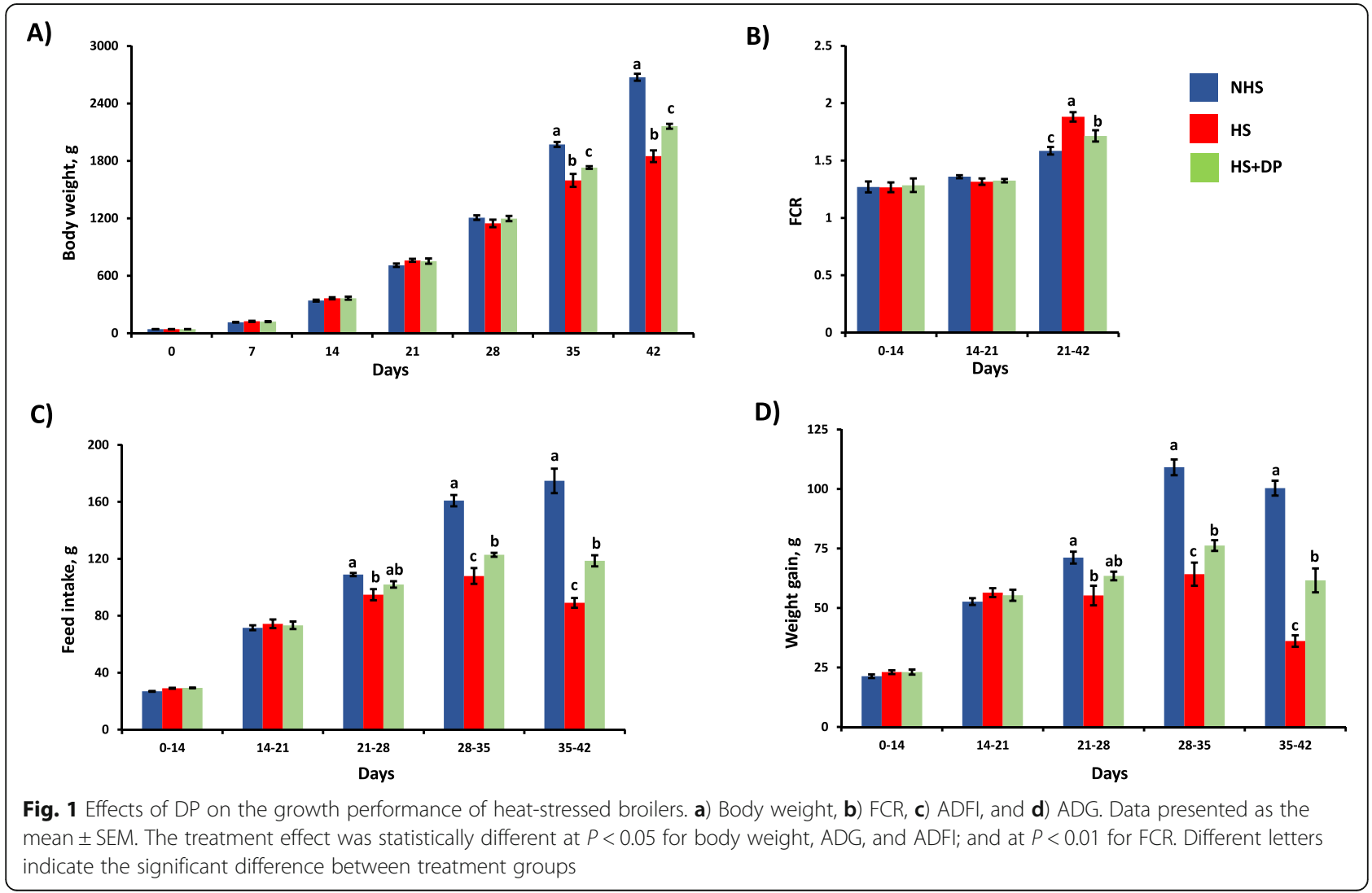


heat-stressed birds as compared to the HS group with basal diet. During the heat stress period (21-42 d), FCR was significantly higher in the birds with normal finisher diet (HS group), while supplementation of DP significantly lowered $(P<0.01)$ FCR in the heat-stressed $(\mathrm{HS}+$ DP) birds.

\section{Effects of dried plum on the intestinal gene expression}

The expression of the heat shock protein-related genes (HSF1, HSF3, HSP70, and HSP90) across the treatment is summarized in Fig. 2a. The expression of HSF1 and HSF3 mRNA was significantly increased $(P<0.05)$ in the heat-stressed birds supplemented with the DP than the HS and NHS groups. The mRNA expression of the HSP9O was significantly decreased $(P<0.05)$ in the HS group than the NHS group, whereas DP supplementation significantly increased $(P<0.05)$ the expression of HSP90 in the heat-stressed birds as compared to the HS group. The mRNA expressions of the HSP70 in the NHS and HS groups were similar; however, the expression was significantly increased $(P<0.05)$ in the heat-stressed birds supplemented with the DP than the NHS and HS groups.
The expression of the tight-junction-related genes (OCLN and CLDN1) across the treatment is summarized in Fig. 2b. The mRNA expression of OCLN and CLDN1 was significantly increased $(P<0.05) \mathrm{HS}+\mathrm{DP}$ group as compared to the HS group. The expression of OCLN and $C L D N 1$ remained unchanged $(P>0.05)$ between the NHS and HS group; however, the expression of these genes was numerically lower in the HS group than the NHS group.

The expression profile of the antioxidant-related genes is presented in Fig. 2c. The dietary supplementation of DP significantly increased $(P<0.05)$ the expression of SOD1, SOD2, GPX1, GPX3, PRDX1, TXN, and NRF2 in the heat-stressed broilers birds as compared to the HS group. In contrast, the expressions of these genes were insignificant $(P>0.05)$ between the NHS and HS groups.

The expression of immune-related genes (IL4 and $M U C 2)$ across the treatment is summarized in Fig. $2 \mathrm{~d}$. The mRNA expression of the IL4 was significantly decreased $(P<0.05)$ in the HS group as compared to the NHS group, while supplementation of DP significantly increased $(P<0.05)$ the expression of IL4 in heatstressed birds than the HS group. Supplementation of

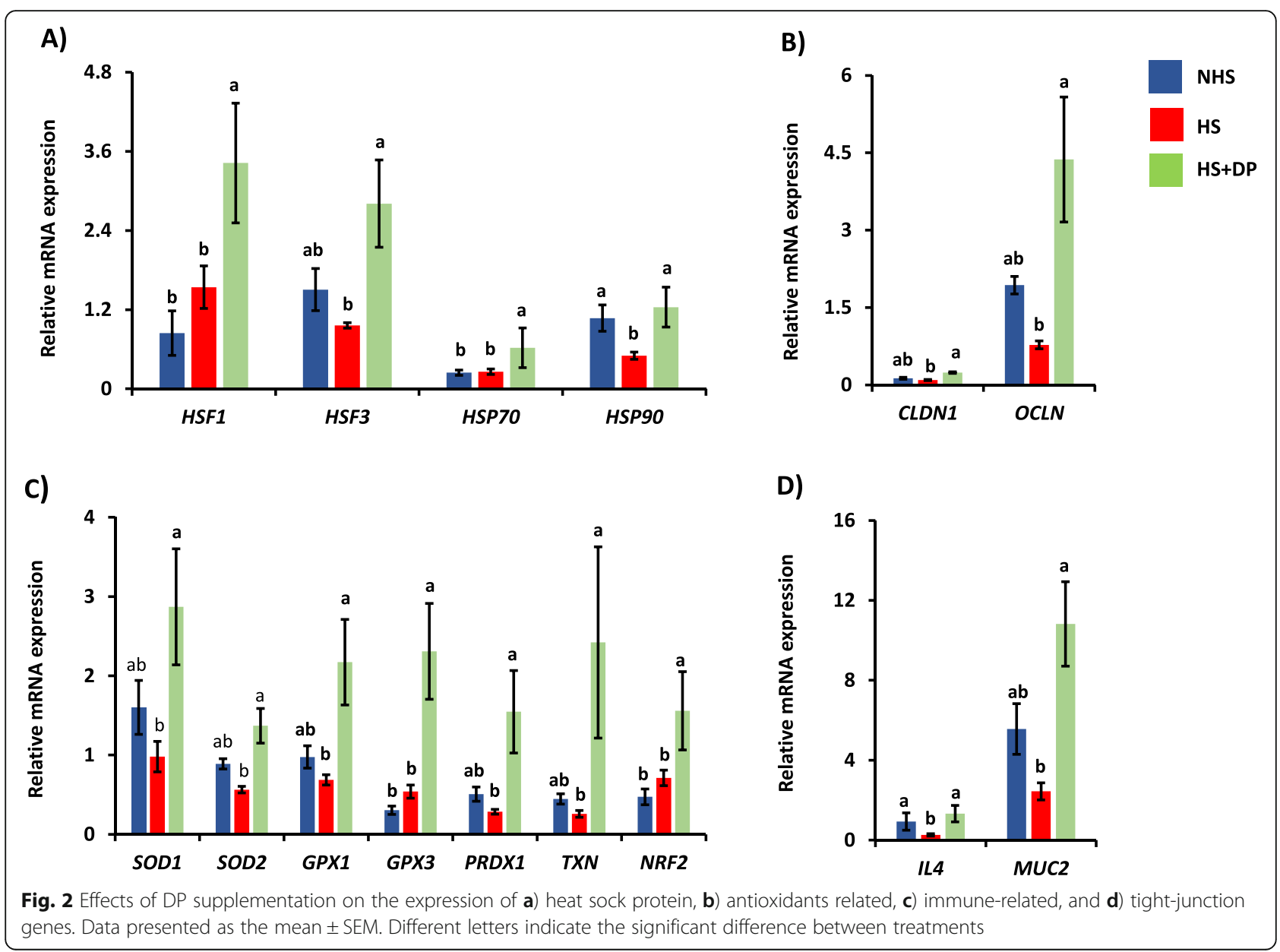


DP in the heat-stressed birds also significantly increased $(P<0.05)$ mRNA expression of the MUC2 than the HS group. Although mRNA expression of the $M U C 2$ was relatively lower in the HS group than the NHS group, there was no significant difference $(P>0.05)$ in the expression of this gene in these two groups.

\section{lleum histomorphology}

The villus height, and villus height to crypt depth ratio were significantly decreased $(P<0.05)$ in the HS group than the NHS group, while supplementing DP in heatstressed birds exhibited improvement in these parameters, but statistically not significant (Fig. 3). The villus surface area was significantly decreased $(P<0.05)$ in the HS group as compared to the NHS group. Dietary supplementation of DP did not exhibit improvement for the surface area in heat-stressed birds. No significant changes $(P>0.05)$ were observed for the crypt depth.

\section{Volatile fatty acids (VFA)}

The concentration of propionate was significantly decreased $(P<0.05)$ in HS groups than the NHS groups, while dietary supplementation of the DP significantly increased $(P<0.05)$ its amount in the cecal digesta as compared to the HS group (Fig. 4). Dietary supplementation of the DP also significantly increased $(P<0.05)$ the acetate concentration in the heat-stressed birds than the HS group. There was no significant difference $(P>0.05)$ in the butyrate concentration between treatment groups. Overall, the DP supplemented group (HS + DP) exhibited a significant increase in the concentration of total VFAs compared to the HS group.

\section{Alpha and beta diversity of cecal microbiota}

Alpha diversity measured the variance (diversity) within a treatment and was measured by Shannon entropy and Simpson's index in this study (Fig. 5). Simpson's index measures dominance or evenness while the Shannon entropy considers both the species richness and the community's evenness. In this study, both Shannon and Simpson's index significantly increased in the HS + DP group compared to the NHS group.

Beta diversity measured the difference in the microbial composition between different environments (treatment groups) and was determined by unweighted UniFrac, and weighted UniFrac (Fig. 6). The unweighted UniFrac based PCoA reveals a significant difference in microbial composition between the treatment groups (PERM ANOVA analysis, $P$-value $=0.00181$.

\section{Cecal microbial composition}

The cecal microbiota composition at the phylum level between treatment groups after removing OTUs with low abundance is shown in Fig. 7a. Firmicutes and Bacteroidetes were the major dominant phyla across the samples in the NHS (49\% and 50\%, respectively), HS (62\% and $36 \%$, respectively), and HS + DP (62\% and 35\%, respectively).

At the class level (Fig. 7b), the cecal microbiota of the broiler birds in the NHS was dominated by the Bacteroidia (50.17\%) followed by Clostridia (47.17\%), while HS and HS + DP groups were dominated by Clostridia $(61.83 \%$ and $60.67 \%)$, followed by Bacteroidia (35.83\% and $34.167 \%)$.

At the order level (Fig. 7c), Bacteroidales was dominated in the NHS group followed by Clostridiales, whereas Clostridiales was predominant in $\mathrm{HS}$ and $\mathrm{HS}+$

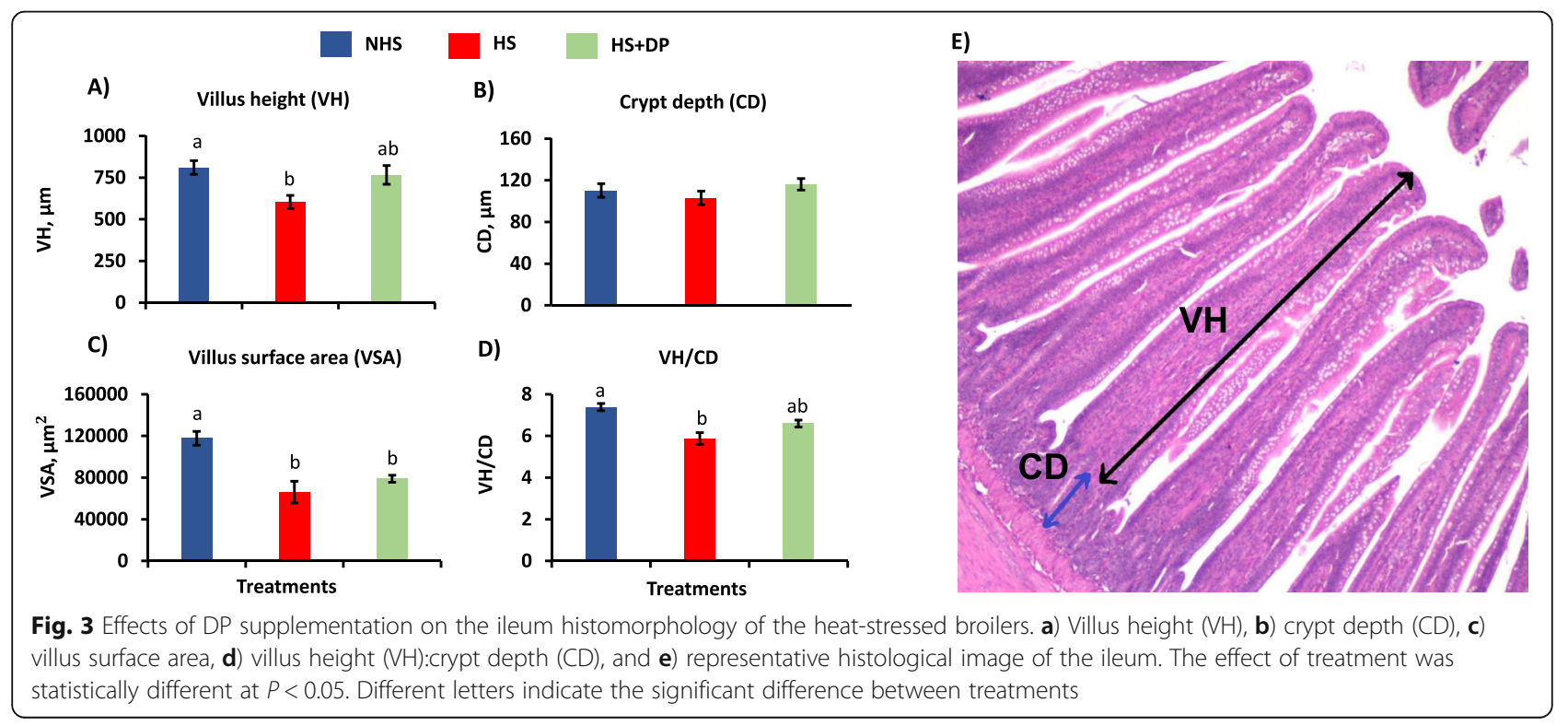


NHS

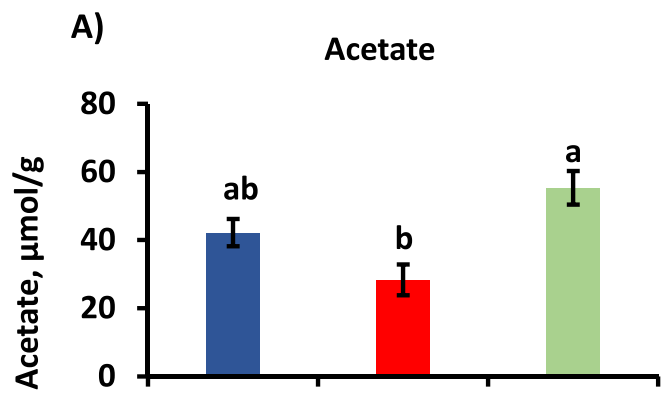

C)

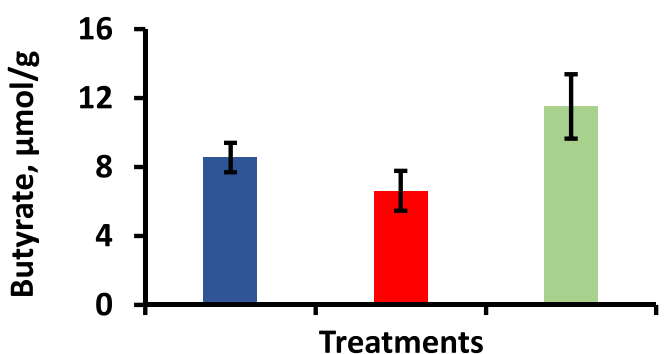

HS

HS+DP

B)

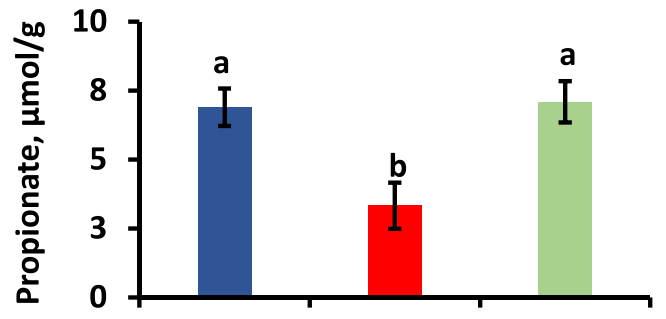

D)

Total VFA

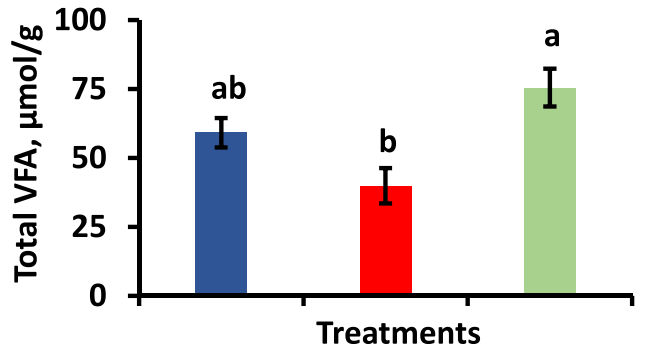

Fig. 4 Effects of DP supplementation on the major volatile fatty acids in the cecal digesta of the heat-stressed broilers. a) Acetate, b) Propionate, c) Butyrate, and $\mathbf{d}$ ) Total VFA. The effect of treatment was statistically different at $P<0.05$. Different letters indicate the significant difference between treatments

DP group followed by Bacteroidales. However, the relative abundance of these dominant taxa was not significant across different groups. However, at the order level, Bacillales was significantly abundant $(P<0.05)$ in the HS + DP group compared to the HS group.

The families Porphyromonadaceae, Ruminococcaceae, and Lachnospiraceae were major dominant families in NHS, HS, and HS + DP groups (Fig. 8). However, these families were not significantly different across different groups. However, at the family level, Bacillaceae, Christensenellaceae, and Peptostreptococcaceae were significantly enriched $(P<0.05)$ in the HS + DP group as compared to the HS group.

Taxon-based analysis at the genus level revealed Parabacteroides, \{Unknown Family\} Clostridiales, and Oscillospira as the predominant genera (Fig. 9). The relative abundance of these genera was not statistically different across different groups. The unknown genus of Bacillaceae, Anaerotruncus, unknown genus of Christensenellaceae, and unknown genus of Peptostreptococcaceae were significantly enriched $(P<0.05)$ in the HS + DP group at the genus level as compared to the HS group.
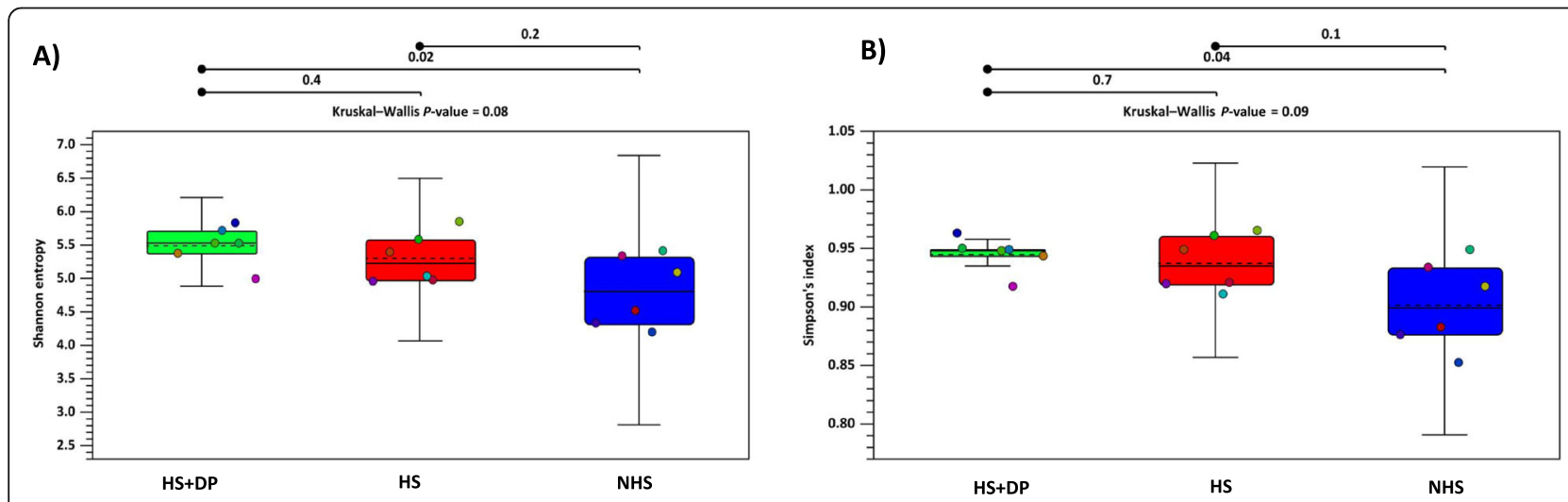

Fig. 5 Effects of DP supplementation on microbial alpha diversity in heat-stressed birds. a) Shannon entropy and b) Simpson's index 


\section{A) weighted UniFrac}
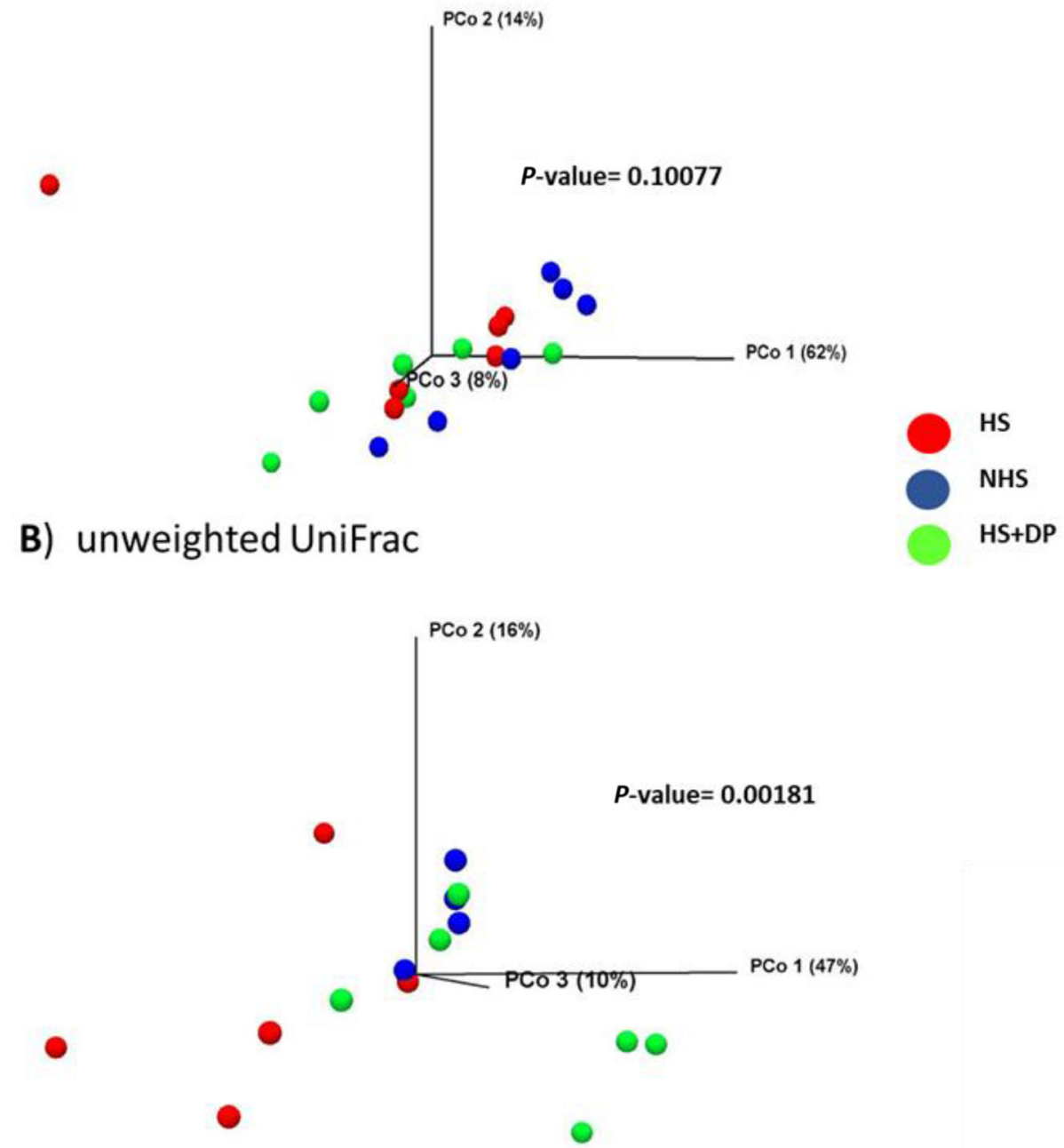

Fig. 6 Effects of DP supplementation on microbial beta diversity in heat-stressed broilers. a) Weighted UniFrac and b) Unweighted UniFrac

Correlation between the differential microbial species and measured parameters

Spearman's rank correlation was calculated to identify the association between the bacterial taxa and the changed parameters considered in this study. Significant correlations observed are shown in Table 2 . The antioxidant genes (SOD2, TXN, and PRDX1) were positively associated with order Bacillales, families Bacillaceae and Christensenellaceae, and genus Anaerotruncus. The GPX1 was positively associated with order Bacillales, family Bacillaceae and genus Anaerotruncus; GPX3 and CLAU1 were positively associated with order Bacillales, families Bacillaceae and Peptostreptococcaceae, and genus Anaerotruncus; NRF2 was positively associated with families Christensenellaceae and Peptostreptococcaceae; HSF3 and IL4 were positively associated with genus Anaerotruncus; and MUC2 and OCLN were positively associated with order Bacillales, family Bacillaceae and genus Anaerotruncus. For VFA, Acetate was positively associated with the family Christensenellaceae and genus Anaerotruncus. While total VFAs were positively associated with order Bacillales, families Bacillaceae and Christensenellaceae, and genus Anaerotruncus.

\section{Discussion}

Environmental heat stress negatively impacts broiler chickens' growth performances by inducing oxidative stress and impairing the physiological parameters $[2,18$, 19]. In poultry, feed intake reduces by $5 \%$ for every $1{ }^{\circ} \mathrm{C}$ rise in the temperature range of $32-38^{\circ} \mathrm{C}$ [20]. In this study, heat stress significantly decreased the final body weight, ADFI, ADG, FCR, and gut health parameters. 

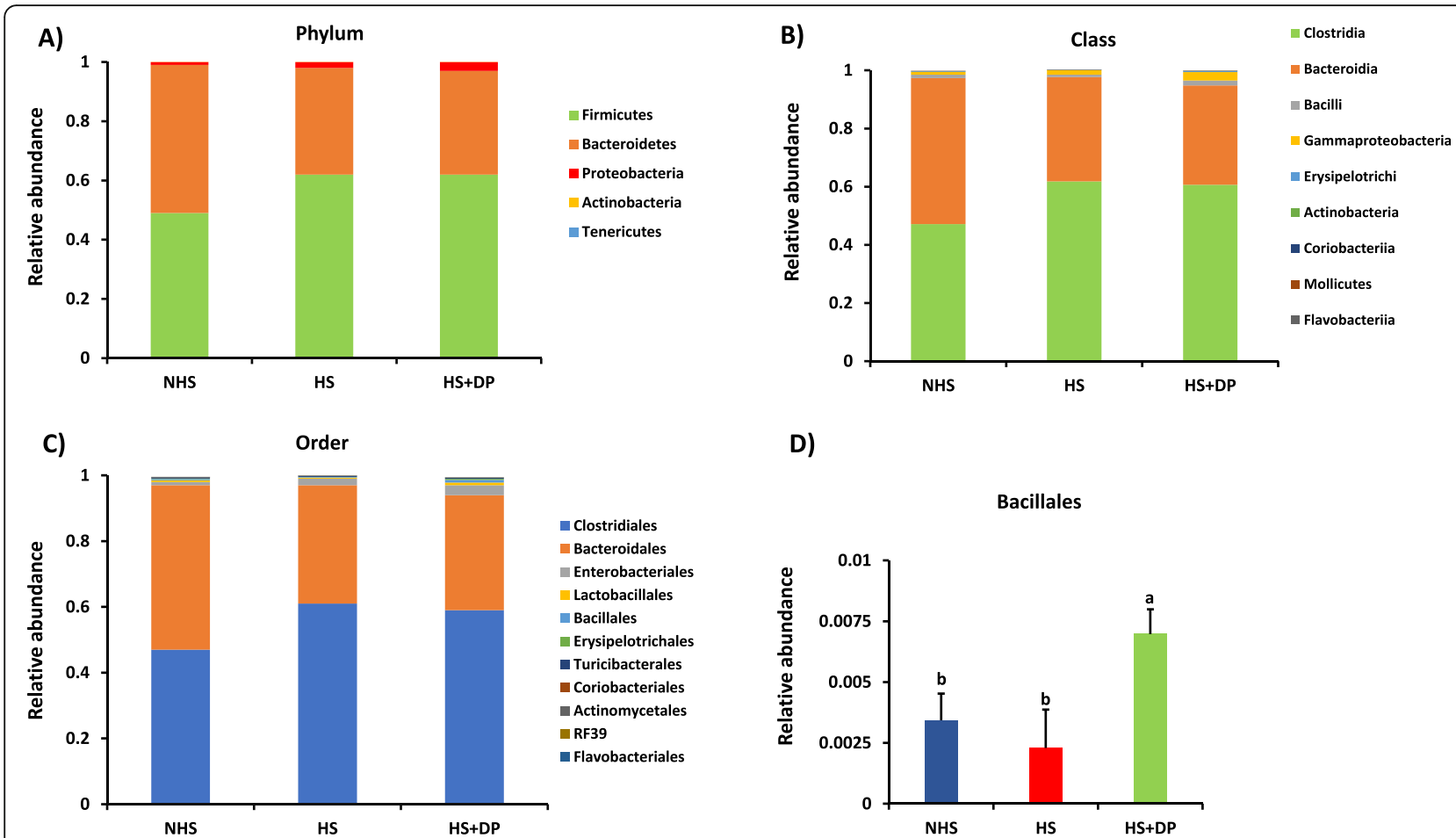

D)

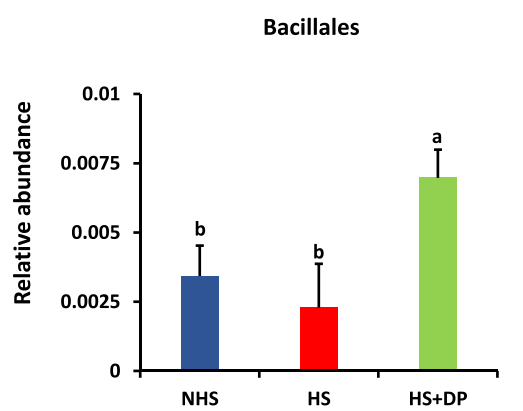

Fig. 7 Average relative abundance of the microbiota at the phylum (a), class (b), order level (c), and significantly abundance microbiota at the order level (d)

The dietary supplementing DP (2.5\%), on the other hand, improved the growth performance (body weight, ADG, ADFI, and FCR) along with the expression of heat shock protein-related, antioxidant related, immunerelated, and tight-junction related genes in the heatstressed birds. Besides, DP supplementation also improved intestinal integrity, increased butyrate along with total VFAs concentration in the cecum, and enriched the relative abundance of beneficial bacteria in the cecum. Improvement in the production performance while fortifying the diet with $2.5 \%$ DP can be attributed to antioxidants, polyphenols, vitamins, and minerals. Similarly, FCR in heat-stressed birds supplemented with DP was improved, which can be ascribed to a better weight gain and feed utilization.

The gastrointestinal tract (GIT) is considered as the main target of heat stress [21]. To delineate the potential mechanism by which DP improved the growth performances of heat-stressed birds, different gut health parameters were analyzed. The ileum, the terminal part of the small intestine, is associated with the absorption of most of the nutrients in the poultry and is more prone to heat stress [22]. In response to the elevated temperature, the cell possesses two major kinds of protective mechanisms to maintain normal cellular functions: firstly, by the production of the HSPs, and secondly, by increasing the production of the antioxidants inside the cell. The HSPs are a group of proteins that are produced by the cell under stress conditions. These proteins are transcriptionally regulated by heat shock factors (HSFs) [23]. Both HSP70 and HSP90 acts as chaperons, ensure proper folding of the proteins, and have cytoprotective action [24]. Besides this, an increased expression of HSP7O was increased in broilers digestive enzyme activity [25] and promote the production of glutathione (GSH), superoxide dismutase (SOD), and total antioxidant capacity (TAOC) [26]. Previous studies have reported that HSFs and HSPs are upregulated during acute heat stress [22, 27]. In this study, however, the chickens subjected to heat stress had significantly lower expression of HSF3 and HSP90. The expressions of HSFs and HSPs are found to be tissuespecific and varied with the stress duration (i.e., acute vs. chronic stress) [28]. Moreover, the biphasic expression pattern of HSP70s was observed in the cattle [29]. Therefore, spatiotemporal expressions of these HSFs and HSPs are required to understand the functionality of these molecules. Interestingly, the expression of HSF 1 , HSF3, HSF70, and HSP9O were increased in heatstressed birds supplemented with DP. The increased expression of $H S P 70$ and $H S P 90$ is likely due to the increased expression of HSF1 and HSF3. The role of HSF1 in the induction of HSP70 [30], and HSF3 in promoting the expression of all HSPs [31] in the chicken are widely 

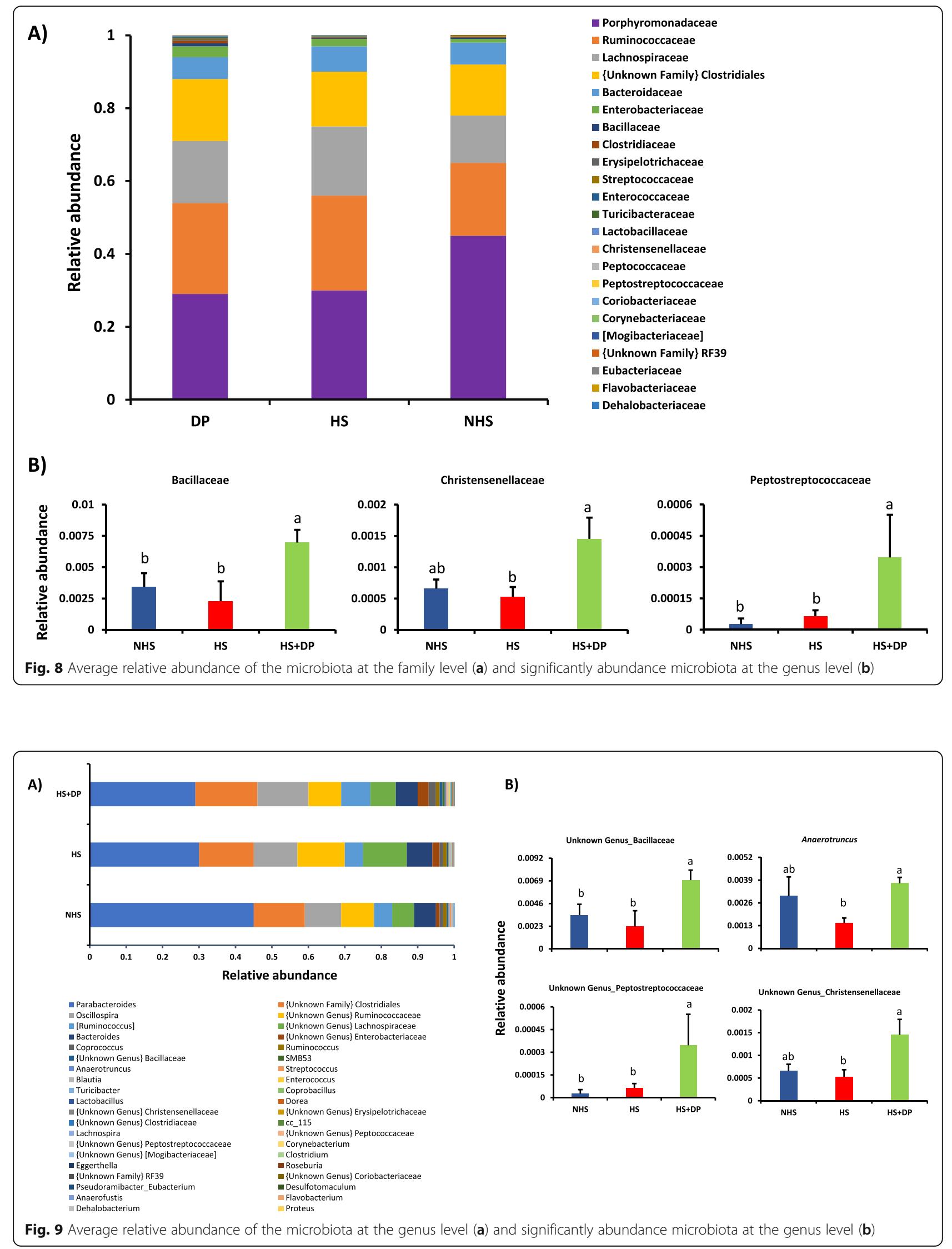
Table 2 Spearman correlation between the differential microbial species and changed measure parameters

\begin{tabular}{|c|c|c|c|}
\hline Variables & Differential microbial species & Spearman $\rho$ & Prob $>\rho$ \\
\hline SOD2 & $\{$ Unknown Genus\} Bacillaceae & 0.4876 & $0.0401^{*}$ \\
\hline SOD2 & Anaerotruncus & 0.6367 & $0.0045^{*}$ \\
\hline SOD2 & $\{$ Unknown Genus\} Christensenellaceae & 0.5046 & $0.0327^{*}$ \\
\hline SOD2 & O_Bacillales & 0.4876 & $0.0401^{*}$ \\
\hline SOD2 & F_Bacillaceae & 0.4876 & $0.0401^{*}$ \\
\hline SOD2 & F_Christensenellaceae & 0.5046 & $0.0327^{*}$ \\
\hline GPX1 & $\{$ Unknown Genus\} Bacillaceae & 0.4721 & $0.0479^{*}$ \\
\hline GPX1 & Anaerotruncus & 0.5521 & $0.0175^{*}$ \\
\hline GPX1 & Bacillales_order & 0.4721 & $0.0479^{*}$ \\
\hline GPX1 & F_Bacillaceae & 0.4721 & $0.0479^{*}$ \\
\hline GPX3 & $\{$ Unknown Genus\} Bacillaceae & 0.4928 & $0.0377^{*}$ \\
\hline GPX3 & Anaerotruncus & 0.4716 & $0.0482^{*}$ \\
\hline GPX3 & $\{$ Unknown Genus\} Peptostreptococcaceae & 0.514 & $0.0291^{*}$ \\
\hline GPX3 & O_Bacillales & 0.4928 & $0.0377^{*}$ \\
\hline GPX3 & F_Bacillaceae & 0.4928 & $0.0377^{*}$ \\
\hline GPX3 & F_Peptostreptococcaceae & 0.514 & $0.0291^{*}$ \\
\hline PRDX1 & $\{$ Unknown Genus\} Bacillaceae & 0.4979 & $0.0355^{*}$ \\
\hline PRDX1 & Anaerotruncus & 0.5893 & $0.0101^{*}$ \\
\hline PRDX1 & $\{$ Unknown Genus\} Christensenellaceae & 0.4696 & $0.0493^{*}$ \\
\hline PRDX1 & O_Bacillales & 0.4979 & $0.0355^{*}$ \\
\hline PRDX1 & F_Bacillaceae & 0.4979 & $0.0355^{*}$ \\
\hline PRDX1 & F_Christensenellaceae & 0.4696 & $0.0493^{*}$ \\
\hline TXN & $\{$ Unknown Genus\} Bacillaceae & 0.4928 & $0.0377^{*}$ \\
\hline TXN & Anaerotruncus & 0.5686 & $0.0138^{*}$ \\
\hline TXN & $\{$ Unknown Genus\} Christensenellaceae & 0.5666 & $0.0142^{*}$ \\
\hline TXN & O_Bacillales & 0.4928 & $0.0377^{*}$ \\
\hline TXN & F_Bacillaceae & 0.4928 & $0.0377^{*}$ \\
\hline TXN & F_Christensenellaceae & 0.5666 & $0.0142^{*}$ \\
\hline NRF2 & \{Unknown Genus\} Christensenellaceae & 0.4951 & $0.0433^{*}$ \\
\hline NRF2 & $\{$ Unknown Genus\} Peptostreptococcaceae & 0.5029 & $0.0396^{*}$ \\
\hline NRF2 & F_Christensenellaceae & 0.4951 & $0.0433^{*}$ \\
\hline NRF2 & F_Peptostreptococcaceae & 0.5029 & $0.0396^{*}$ \\
\hline HSF3 & Anaerotruncus & 0.4757 & $0.0460^{*}$ \\
\hline CLDN1 & $\{$ Unknown Genus\} Bacillaceae & 0.5196 & $0.0271^{*}$ \\
\hline CLDN1 & Anaerotruncus & 0.6739 & $0.0022^{*}$ \\
\hline CLDN1 & $\{$ Unknown Genus\} Peptostreptococcaceae & 0.6004 & $0.0084^{*}$ \\
\hline CLDN1 & O_Bacillales & 0.5196 & $0.0271^{*}$ \\
\hline CLDN1 & F_Bacillaceae & 0.5196 & $0.0271^{*}$ \\
\hline CLDN1 & F_Peptostreptococcaceae & 0.6004 & $0.0084^{*}$ \\
\hline OCLN & $\{$ Unknown Genus\} Bacillaceae & 0.5134 & $0.0293^{*}$ \\
\hline OCLN & Anaerotruncus & 0.7172 & $0.0008^{*}$ \\
\hline OCLN & O_Bacillales & 0.5134 & $0.0293^{*}$ \\
\hline OCLN & F_Bacillaceae & 0.5134 & $0.0293^{*}$ \\
\hline MUC2 & $\{$ Unknown Genus\} Bacillaceae & 0.5785 & $0.0119^{*}$ \\
\hline
\end{tabular}


Table 2 Spearman correlation between the differential microbial species and changed measure parameters (Continued)

\begin{tabular}{llll}
\hline Variables & Differential microbial species & Spearman $\boldsymbol{\rho}$ & Prob $>\boldsymbol{\rho}$ \\
\hline MUC2 & Anaerotruncus & 0.5377 & $0.0214^{*}$ \\
MUC2 & Bacillales_order & 0.5785 & $0.0119^{*}$ \\
MUC2 & F_Bacillaceae & 0.5785 & $0.0119^{*}$ \\
IL4 & Anaerotruncus & 0.4737 & $0.0471^{*}$ \\
Acetate & Anaerotruncus & 0.4964 & $0.0361^{*}$ \\
Acetate & $\{$ Unknown Genus\} Christensenellaceae & 0.6512 & $0.0034^{*}$ \\
Acetate & F_Christensenellaceae & 0.6512 & $0.0034^{*}$ \\
Total VFA & $\{$ Unknown Genus\} Bacillaceae & 0.4731 & $0.0474^{*}$ \\
Total VFA & Anaerotruncus & 0.4757 & $0.0460^{*}$ \\
Total VFA & $\{$ Unknown Genus\} Christensenellaceae & 0.5955 & $0.0091^{*}$ \\
Total VFA & O_Bacillales & 0.4731 & $0.0474^{*}$ \\
Total VFA & F_Bacillaceae & 0.4731 & $0.0474^{*}$ \\
Total VFA & F_Christensenellaceae & 0.5955 & $0.0091^{*}$ \\
\hline
\end{tabular}

known. The correlation analysis showed that the expression of HSF3 was positively associated with cecal bacteria Anaerotruncus. This relationship warrants further investigation.

In response to the oxidative stress elicited by heat stress in poultry, Nrf2-a redox-sensitive nuclear transcriptional factor-is translocated in the nucleus, where it binds in the promotor region of the antioxidant response element in the DNA, leading to the production of different antioxidants [32]. Therefore, to better understand the antioxidant status of the heat-stressed birds, different antioxidant related genes such as $S O D 1, S O D 2$, GPX1, GPX3, PRDX1, TXN, and NRF2 were analyzed. SOD1 and SOD2 are isoforms of superoxide dismutase (SOD). SOD1, cytosolic $\mathrm{Cu} / \mathrm{ZnSOD}$, is mainly localized in the cytoplasm, mitochondrial intermembrane spaces, nucleus, lysosomes, and peroxisomes, whereas SOD2 is mitochondrial manganese $(\mathrm{Mn})$ containing enzymes [33]. Superoxide radical is the predominant free radicals produced inside the cell [34]. SODs catalyze the superoxide radical into hydrogen peroxide; thus, it is considered the main element of the first level of antioxidant defense in the cells [35]. Similarly, GPX1 and GPX3 are the selenium-dependent forms of glutathione in avian species. GPX1 is mainly localized in the cytoplasm and mitochondria, while GPX3 is most abundant in the plasma. GPXs catalyzes the reduction of hydroperoxides and $\mathrm{H}_{2} \mathrm{O}_{2}$ by glutathione [36]. PRDX1 is a member of the Peroxiredoxins family, which uses thioredoxin to reduce $\mathrm{H}_{2} \mathrm{O}_{2}$, hydroperoxides, and proximities to balance ROS inside the cell [37]. TXN is a key member of the thioredoxin system-important antioxidant system in defense against oxidative stress in the cell. Moreover, thioredoxin is also involved in immune response, DNA and protein repairs, and cell death [38]. In this study, the expression of NRF2, SOD1, SOD2, GPX1, GPX3, $T X N$, and PRDXN were significantly improved in the heat-stressed birds supplemented with the DP. Indeed, significant expression of the NRF2 along with antioxidants (SOD1, SOD2, GPX1, and GPX3) indicate that DP was able to activate Nrf2 mediated antioxidants enzymes in the heat-stressed birds. Previous experiments on phytochemicals have demonstrated a vital role of polyphenols in the activation of the Nrf2 mediated antioxidants enzymes $[6,39]$. In agreement with those studies, polyphenols present in the DP possibly may have played a similar role resulting in the upregulation of antioxidant genes in heat-stressed birds, thus reducing ROS and lipid peroxidation within the cell.

The intestinal epithelial tight junctional barrier plays a vital role against paracellular penetration of the pathogenic bacteria, endotoxins, and feeds associated antigens [40-42]. Occludin (OCLN) and claudin (CLDN) are the major transmembrane proteins that make up the tight junctions. Occludin helps regulate paracellular permeability and plays a key role in cellular structure and barrier function. Claudins form the backbone of tight junctions and play a significant role in the tight junction ability to seal the paracellular space [43]. During heat stress, peripheral circulation of the blood increased as a result of blood flow in the epithelium in the intestine is reduced, resulting in hypoxia, which leads to the disruption of tight junction, reduced intestinal integrity, and increases intestinal permeability that is found to increase the circulating endotoxins [44]. In this study, the expression of the OCLN and CLDN1 was significantly increased in the ileum of heat-stressed birds supplemented with DP. This result indicates that dietary DP improved the intestinal tight-junction related genes in the heatstressed birds, which may be attributed as one of the 
reasons for the improvement in the production performance in heat-stressed birds. Flavonoids, polyphenol compounds, exhibit promotive and protective effects on intestinal tight junction barrier functions [45]. Improved expression of the tight junction in the heat stress in the DP supplemented birds may be due to its flavonoids. Besides, Spearman correlation revealed the positive association of OCLN with Bacillales, Bacillaceae and Anaerotruncus; and CLDN1 with Bacillales, Bacillaceae, Peptostreptococcaceae, and Anaerotruncus.

To delineate the effects of DP on the intestinal immune system, the different immune-related genes in the ileum broilers were analyzed. The expression of IL4 and $M U C 2$ was significantly increased by supplementing DP. IL4 is a cytokine that plays a vital role in regulating the immune system and cellular homeostasis [46]. Spearman correlation analysis revealed a positive association of IL4 with Anaerotruncus. Thus, a significant expression of IL4 indicates the enhanced immune response in heatstressed birds. Mucin is the major constituent of mucus layers and plays a pivotal role in protecting the gut from pathogens, acidic chyme, and digestive enzymes, influencing nutrient absorption and digestion [47]. The significant expression of $M U C 2$ has probably played a protective role against pathogens along with enhancing nutrient digestion in heat-stressed broilers.

High temperature has been shown to impair the intestinal integrity in the broilers $[48,49]$. Consistent with those findings, the result of this study demonstrated that the morphology of the ileum was damaged with heat stress, which could be due to the epithelial shedding in response to intestinal ischemia [50]. Although statistically not significant, supplementing DP improved the villus height and villus height to crypt depth ratio in heat-stressed birds. VFAs are found to exert tropic effects on intestinal morphology [51]. In this regard, the beneficial effect of DP on the intestinal morphology may be exhibited due to increased colonization of the beneficial bacteria along with the production of higher amounts of VFAs in heat-stressed birds.

Acetate, propionate, and butyrate are the major VFA produced by the fermentation of the dietary fibers in the poultry's cecum. VFAs are found to play a vital role in the gut and immune homeostasis. Acetate, dominant VFA, is involved in the muscles' glycolytic pathway; propionate is associated with gluconeogenesis in the liver, while the butyrate serves as a fuel for colonocytes [52]. In this study, the amount of acetate, propionate, and total VFA was increased significantly in the heat-stressed birds supplemented with the DP, while the amount of the butyrate was found to increase numerically. There was a positive association of acetate with Christensenellaceae and Anaerotruncus; and a positive association of total VFAs with Bacillales, Bacillaceae,
Christensenellaceae, and Anaerotruncus. These improvements in the VFAs can be attributed to the higher level of dietary fibers present in the DP along with the enriched bacteria. Moreover, these results of VFA help corroborate our finding of improved growth performance, ileum histomorphology, and expression of tightjunction genes.

Gut microbiota plays a crucial role in the intestinal health and growth performances of birds. On the other hand, high temperature has been associated with the dysbiosis of the cecal microbiota in poultry. Supplementation of DP significantly improved bacterial richness and diversity of cecal microbiota in heat-stressed birds, which may be attributed due to the high dietary fibers and polyphenols in the DP. The results of this study show that heat stress in broilers exhibited a significant change in unweighted UniFrac measures of beta diversity, which was consistent with the previous study [53] and indicate that bacteria having relatively lower abundance were significantly different between the treatments.

Afterward, significantly abundant taxa were determined. At the order level-Bacaillale, at the family level-Bacillaceae, Christensenellaceae, Peptostreptococcaceae, and at the genus level - unknown genus of Bacillaceae, Anaerotruncus, unknown genus of Christensenellaceae, and unknown genus of Peptostreptococcaceae were significantly enriched in the heat-stressed birds supplement with the DP. A positive correlation was observed between Bacillaceae and different observed parameters (TXN, PRDX1, $C L D N 1, O C L N, M U C 2$, and total VFAs). In drosophila larvae, the relative abundance of the Bacillaceae was significantly lower in cancerous larvae than those without the tumor. Furthermore, they hypothesized Bacillaecae could eliminate cancer cells at the beginning of carcinogenesis potentially by stimulating the immune system [54]. Additionally, Bacillus spp. member of Bacillaceae is found to display antimicrobial, antioxidant and immunemodulatory activity in the host and has gained significant attention in the past decade as a potential probiotic [55]. Considering these facts, along with our observed results, we can conclude that significant enrichment of Bacillaceae in heat-stressed broiler birds has played a vital role in improving the immune status by enhancing different antioxidants, tight-junction genes, and VFAs.

The family Christensenellaceae belongs to the phylum Firmicutes and plays a vital role in human health [56]. The positive association of Christensenellaceae with antioxidant-related genes (TXN and NRF2), acetate, and total VFAs was observed in our study. Christensenellaceae is considered one of the signature taxa of a healthy gut and are depleted in conditions associated with inflammation [57]. Moreover, the significant abundance of the Christensenellaceae was found to have a negative 
correlation with the visceral fat mass [58] and is also negatively associated with the total cholesterol and lowdensity cholesterol (bad cholesterol). Interestingly, a higher amount of abdominal fat is found in heat-stressed poultry [59]. Taken together, our study showed a significant abundance of the Christensenellaceae in the DP, demonstrates its beneficial effect on the gut. Christensenellaceae are associated with diets higher in fibers [56]. Thus, we can speculate that the significant abundance of the Christensenellaceae in heat-stressed broiler birds supplemented with DP is due to its higher dietary fiber content.

The family Peptostreptococcaceae belongs to phylum Firmicutes and is found as the normal commensal of the gut. They are reported to be higher in the gut of healthy rats than one with dysbiosis [60]. This highlights its role in maintaining gut homeostasis. Studies have shown that Peptostreptococcaceae are involved in the production of VFAs from amino acids. Thus, the significant abundance of the Peptostreptococcaceae may also have involved in increasing the production of VFAs in the ileum of heat-stressed birds supplemented with DP [61].

Finally, at the genus level, Anaerotruncus was a significant enrichment in heat-stressed birds supplemented with DP. The Anaerotruncus was positively associated with IL4, HSF3, CLAU1, OCLN, MUC2, acetate, and total VFA in our study. Interestingly, Anaerotruncus is among the 17 strains of the intestinal bacteria found to stimulate the regulatory $\mathrm{T}$-cells and attenuate the inflammation in a mouse colitis model [62]. Also, antibiotic-induced noninfectious colitis in humans is reported to be treated by the community of 17 intestinal bacteria that include Anaerotruncus by fecal transplantation [63]. Moreover, probiotic supplementation during the recovery phase after antibiotic administration was found to suppress the growth of Shigella and Escherichia, while blooming Anaerotruncus spp. [64]. Considering these, the significant abundance of Anaerotruncus in our study highlights its potential role in attenuating inflammation and enhancing immunity in the intestine.

There have been several studies highlighting the role of vitamins (Vit A, E, and C) and minerals (Mn, $\mathrm{Se}, \mathrm{Zn}, \mathrm{Mg}$, and $\mathrm{Cu}$ ) in mitigating heat stress in poultry [18]. DP is a mixture of bioactive compounds - vitamins, minerals, and polyphenols [9]. A simple calculation shows that the supplementation provides $0.15 \mathrm{mg}$ Vit C, $0.108 \mathrm{mg}$ Vit E, $0.975 \mu \mathrm{g}$ Vit A, 0.0.0748 $\mathrm{mg} \mathrm{Mn}, 0.075 \mu \mathrm{g}$ Se, $0.11 \mathrm{mg} \mathrm{Zn}$, and 10.25 $\mathrm{mg} \mathrm{Mg}$ per $\mathrm{kg}$ of the experimental diet fed to the chicken. Then on top levels provided to the premix, DP is the likely reason for the DP's beneficial effects in the heat-stressed broiler birds. Moreover, higher amounts of glucose obtained from the DP diet also attributed the beneficial effects in the heat-stressed broiler birds. Further nutrient profiling of DP will help precise feed formulation and evaluate its additional performance and health benefits in poultry.

\section{Conclusion}

The results of this study revealed that the dietary supplementation of DP significantly improved the body weight, ADG, ADFI, FCR, ileum histomorphology, cecal VFA production, and expression of heat sock, antioxidant, immune, and tight junction related genes in the heatstressed birds. Moreover, DP was also able to improve the relative abundance of beneficial bacteria in the chicken gut. Thus, considering the benefits of supplementing DP in heat-stressed birds, the dietary supplementation of DP can be considered a potential strategy to mitigate the negative effects of heat stress in poultry.

\section{Supplementary Information}

The online version contains supplementary material available at https://doi. org/10.1186/s40104-021-00571-5.

Additional file 1: Table S1. Nutrient composition of dried plum. Table S2. Primers used to 838 quantify the expression of the genes by qPCR. Fig. S1. Experimental design.

\section{Abbreviations \\ ADFI: Average daily feed intake; ADG: Average daily gain; ALA : Alpha-lipoic acid; ANOVA: Analysis of variance; CD: Crypt depth; CDNA: Complementary deoxyribonucleic acid; CLDN1: Claudin 1; DP: Dried plum; FCR: Feed conversion ratio; GC: Gas chromatography; GPX1: Glutathione Peroxidase 1; GPX3: Glutathione Peroxidase 3; HS: Heat stress; HSF1: Heat shock factor 1; HSF3: Heat shock factor 3; HSP70: Heat Shock Protein 70; HSP90: Heat Shock Protein 90; IL4: Interleukin 4; MUC2: Mucin 2; OCLN: Occludin; OTU: Operational taxonomic unit; PCR: Polymerase chain reaction; PRDX1: Peroxiredoxin 1; qPCR: Quantitative polymerase chain reaction; ROS: Reactive oxygen species; SOD1: Superoxide dismutase 1; \\ SOD2: Superoxide dismutase 2; TMA : Trimethyl acetate; TXN: Thioredoxin; VFA: Volatile fatty acid; VH: Villus height}

\section{Acknowledgments}

We sincerely thank Socorro Tauyan for helping in animal experimentation and Dr. Mohammad Arif for providing the facilities for Microbiome Analysis.

\section{Authors' contributions}

S.W. conducted the experiments, collected samples, performed sample analyses, and drafted the manuscript. N.S. helped in animal experimentation, sampling, and manuscript preparation. A.K. S. helped in sampling and VFA analyses. C.N.L. contributed to drafting this manuscript. R.J. helped in feed formulation, data analysis, and manuscript preparation. B.M. designed the experiments, helped in sampling, data interpretation, and manuscript writing. All the authors read and approved the manuscript.

\section{Funding}

This work was supported by a Start-up grant from CTAHR University of Hawaii at Manoa, and USDA Multistate (2052R) to B.M. Apart from providing funds, these organizations were not involved in any experimental procedure and manuscript preparation.

\section{Availability of data and materials}

The sequence data reported in this paper have been deposited in the NCB database (Metagenomic sequencing data: PRJNA688117). 


\section{Ethics approval and consent to participate}

All the animal experimentation was carried out following the guidelines approved by the Institutional Animal Care and Use Committee of the University of Hawaii at Manoa (Approval No.17-2605).

\section{Consent for publication}

Not applicable.

\section{Competing interests}

The authors declare that they have no competing interests.

\section{Received: 17 August 2020 Accepted: 17 February 2021} Published online: 30 March 2021

\section{References}

1. St-Pierre NR, Cobanov B, Schnitkey G. Economic losses from heat stress by US livestock industries. J Dairy Sci. 2003;86:E52-77.

2. Wasti S, Sah N, Mishra B. Impact of heat stress on poultry health and performances, and potential mitigation strategies. Animals. 2020;10(8):1-19.

3. Zuidhof MJ, Schneider BL, Carney VL, Korver DR, Robinson FE. Growth, efficiency, and yield of commercial broilers from 1957, 1978, and 2005. Poult Sci. 2014;93:2970-82.

4. Tallentire CW, Leinonen I, Kyriazakis I. Breeding for efficiency in the broiler chicken: a review. Agron Sustain Dev Springer-Verlag France. 2016;36(4):66.

5. Lin H, Jiao HC, Buyse J, Decuypere E. Strategies for preventing heat stress in poultry. Worlds Poult Sci J. 2006:62:71-86.

6. Hu R, He Y, Arowolo MA, Wu S, He J. Polyphenols as potential attenuators of heat stress in poultry production. Antioxidants. 2019;8(3):67.

7. Schreurs AS, Shirazi-Fard Y, Shahnazari M, Alwood JS, Truong TA, Tahimic CGT, et al. Dried plum diet protects from bone loss caused by ionizing radiation. Sci Rep. 2016;6:1-11.

8. Castaldi P, Degen J. M. Dried Plums : Natural Solutions to Improve the Quality, Nutrition and Safety of Foods in Space Travel. SAE Tech Pap. 2003; No. 2003-01-2377.

9. Stacewicz-Sapuntzakis M. Dried plums and their products : composition and health effects - an updated review dried plums and their products : composition and health effects. Crit Rev Food Sci Nutr. 2013:53:1277-302.

10. Arjmandi BH, Johnson SA, Pourafshar S, Navaei N. Bone-protective effects of dried plum in postmenopausal women : Efficacy and.possible mechanisms. Nutrients. 2017;9(5):496.

11. Bu SY, Lerner M, Stoecker BJ, Boldrin E, Brackett DJ, Lucas EA, et al. Dried plum polyphenols inhibit osteoclastogenesis by downregulating NFATC1 and inflammatory mediators. Calcif Tissue Int. 2008;82(6):475-88.

12. Hooshmand S, Kumar A, Zhang J, Johnson SA, Chai SC, Arjmandi BH. Evidence for anti-inflammatory and antioxidative properties of dried plum polyphenols in macrophage RAW 264.7 cells. Food Funct. 2015;6(5):1719-25.

13. NRC. Nutrient Requirements of Poultry. Ninth Revi. Washington, DC: National Academies Press; 1994.

14. Sah N, Kuehu DL, Khadka VS, Deng Y, Peplowska K, Jha R, et al. RNA sequencing-based analysis of the laying hen uterus revealed the novel genes and biological pathways involved in the eggshell biomineralization. Sci Rep. 2018:8(1):16853.

15. Wasti S, Sah N, Kuehu DL, Kim YS, Jha R, Mishra B. Expression of follistatin is associated with egg formation in the oviduct of laying hens. Anim Sci J. 2020;91(1):e13396.

16. Iji PA, Saki A, Tivey DR. Body and intestinal growth of broiler chicks on a commercial starter diet. 1. Intestinal weight and mucosal development. Br Poult Sci. 2001;42:505-13.

17. Singh AK. In ovo and post-hatch nutritional programming to improve broiler performance and gut health. University of Hawai'i at Manoa; 2019.

18. Emami NK, Jung U, Voy B, Dridi S. Radical response: effects of heat stressinduced oxidative stress on lipid metabolism in the avian liver. Antioxidants (Basel). 2020;10(1):35.

19. Mishra B, Jha R. Oxidative stress in the poultry gut: potential challenges and interventions. Front Vet Sci. 2019;6:60.

20. Naga Raja Kumari K, Narendra Nath D. Ameliorative measures to counter heat stress in poultry. 2018;74(1):117-30.

21. Lambert GP. Stress-induced gastrointestinal barrier dysfunction and its inflammatory effects. J Anim Sci. 2009;87:E101-8.

22. Varasteh S, Braber S, Akbari P, Garssen J, Fink-Gremmels J. Differences in susceptibility to heat stress along the chicken intestine and the protective effects of galacto-oligosaccharides. PLoS One. 2015;10(9): e0138975.

23. Åkerfelt M, Morimoto Rl, Sistonen L. Heat shock factors: integrators of cell stress, development and lifespan. Nat Rev Mol cell Biol. 2010;11(8): 545.

24. Wegele H, Müller L, Buchner J. Hsp70 and Hsp90 — a relay team for protein folding. Rev Physiol Biochem Pharmacol. Berlin, Heidelberg: Springer; 2004. p. 1-44.

25. Hao Y, Gu XH, Wang XL. Overexpression of heat shock protein 70 and its relationship to intestine under acute heat stress in broilers: 1. Intestinal structure and digestive function. Poult Sci. 2012;91(4):781-9.

26. Gu XH, Hao Y, Wang XL. Overexpression of heat shock protein 70 and its relationship to intestine under acute heat stress in broilers: 2 . Intestinal oxidative stress. Poult Sci. 2012;91(4):790-9.

27. Flees J, Rajaei-Sharifabadi H, Greene E, Beer L, Hargis BM, Ellestad L, et al. Effect of Morinda citrifolia (noni)-enriched diet on hepatic heat shock protein and lipid metabolism-related genes in heat stressed broiler chickens. Front Physiol. 2017;8:919.

28. Xie J, Tang L, Lu L, Zhang L, Xi L, Liu HC, et al. Differential expression of heat shock transcription factors and heat shock proteins after acute and chronic heat stress in laying chickens (Gallus gallus). PLoS One. 2014;9.

29. Bharati J, Dangi SS, Chouhan VS, Mishra SR, Bharti MK, Verma V, et al. Expression dynamics of HSP70 during chronic heat stress in Tharparkar cattle. Int J Biometeorol. 2017;61:1017-27.

30. Inouye S, Katsuki K, Izu H, Fujimoto M, Sugahara K, Yamada SI, et al. Activation of heat shock genes is not necessary for protection by heat shock transcription factor 1 against cell death due to a single exposure to high temperatures. Mol Cell Biol. 2003;23(16):5882-95.

31. Tanabe M, Kawazoe Y, Takeda S, Morimoto RI, Nagata K, Nakai A. Disruption of the HSF3 gene results in the severe reduction of heat shock gene expression and loss of thermotolerance. EMBO J. 1998;17(6):1750-8.

32. Surai PF, Kochish II, Fisinin VI, Kidd MT. Antioxidant Defence systems and oxidative stress in poultry biology: an update. Antioxidants. 2019;8(7):235.

33. Fukai T, Ushio-Fukai M. Superoxide dismutases: role in redox signaling, vascular function, and diseases. Antioxid Redox Signal. 2011;15(6):1583-606.

34. Singal PK, Khaper N, Palace V, Kumar D. The role of oxidative stress in the genesis of heart disease. Cardiovasc Res. 1998;40(3):426-32.

35. Surai PF. Antioxidant systems in poultry biology: superoxide dismutase. J Anim Res Nutr. 2016;1(1):8.

36. Surai PF, Kochish II, Fisinin VI. Glutathione peroxidases in poultry biology: part 1. Classification and mechanisms of action. Worlds Poult Sci J. 2018; 74(2):185-98.

37. Neumann CA, Krause DS, Carman CV, Das S, Dubey DP, Abraham JL, et al. Essential role for the peroxiredoxin Prdx1 in erythrocyte antioxidant defence and tumour suppression. Nature. 2003;424(6948):561-5.

38. Lu J, Holmgren A. The thioredoxin antioxidant system. Free Radic Biol Med. 2014;66:75-87.

39. Zhang J, Bai KW, He J, Niu Y, Lu Y, Zhang L, et al. Curcumin attenuates hepatic mitochondrial dysfunction through the maintenance of thiol pool, inhibition of mtDNA damage, and stimulation of the mitochondrial thioredoxin system in heat-stressed broilers. J Anim Sci. 2018;96(3):867-79.

40. Anderson JM, Van Itallie CM. Tight junctions and the molecular basis for regulation of paracellular permeability. Am J Physiol Liver Physiol. 1995; 269(4):G467-75.

41. Dokladny K, Moseley PL, Ma TY. Physiologically relevant increase in temperature causes an increase in intestinal epithelial tight junction permeability. Am J Physiol Liver Physiol. 2006;290(2):G204-12.

42. Moeser AJ, Klok CV, Ryan KA, Wooten JG, Little D, Cook VL, et al. Stress signaling pathways activated by weaning mediate intestinal dysfunction in the pig. Am J Physiol Liver Physiol. 2007;292(1):G173-81.

43. Lee SH. Intestinal permeability regulation by tight junction: implication on inflammatory bowel diseases. Intest Res. 2015;13(1):11.

44. Pearce SC, Mani V, Boddicker RL, Johnson JS, Weber TE, Ross JW, et al. Heat stress reduces intestinal barrier integrity and favors intestinal glucose transport in growing pigs. PLoS One. 2013;8(8):e70215.

45. Suzuki T, Hara H. Role of flavonoids in intestinal tight junction regulation. J Nutr Biochem. 2011;22(5):401-8.

46. Zhu J. T helper 2 (Th2) cell differentiation, type 2 innate lymphoid cell (ILC2) development and regulation of interleukin-4 (IL-4) and IL-13 production. Cytokine. 2015;75(1):14-24. 
47. Montagne L, Piel C, Lalles JP. Effect of diet on mucin kinetics and composition: nutrition and health implications. Nutr Rev. 2004;62(3):105-14.

48. Burkholder KM, Thompson KL, Einstein ME, Applegate TJ, Patterson JA. Influence of stressors on normal intestinal microbiota, intestinal morphology, and susceptibility to Salmonella enteritidis colonization in broilers. Poult Sci. 2008;87(9):1734-41.

49. Sohail MU, Hume ME, Byrd JA, Nisbet DJ, ljaz A, Sohail A, et al. Effect of supplementation of prebiotic mannan-oligosaccharides and probiotic mixture on growth performance of broilers subjected to chronic heat stress. Poult Sci. 2012;91(9):2235-40.

50. Rivera $L R$, Thacker M, Pontell $L$, Cho HJ, Furness JB. Deleterious effects of intestinal ischemia/reperfusion injury in the mouse enteric nervous system are associated with protein nitrosylation. Cell Tissue Res. 2011;344(1):111-23.

51. Wong JM, De Souza R, Kendall CW, Emam A, Jenkins DJ. Colonic health: fermentation and short chain fatty acids. J Clin Gastroenterol. 2006;40(3): 235-43.

52. Jha R, Fouhse JM, Tiwari UP, Li L, Willing BP. Dietary fiber and intestinal health of monogastric animals. Front Vet Sci. 2019;6:48.

53. Xing $S$, Wang $X$, Diao H, Zhang M, Zhou Y, Feng J. Changes in the cecal microbiota of laying hens during heat stress is mainly associated with reduced feed intake. Poult Sci. 2019;98(11):5257-64.

54. Jacqueline C, Brazier L, Faugère D, Renaud F, Thomas F, Roche B. Can intestinal microbiota be associated with non-intestinal cancers? Sci Rep. 2017;7(1):12722

55. Elshaghabee FM, Rokana N, Gulhane RD, Sharma C, Panwar H. Bacillus as potential probiotics: status, concerns, and future perspectives. Front Microbiol. 2017;8:1490.

56. Waters $\mathrm{JL}$, Ley RE. The human gut bacteria Christensenellaceae are widespread, heritable, and associated with health. BMC Biol. 2019;17(1):83.

57. Mancabelli L, Milani C, Lugli GA, Turroni F, Cocconi D, Van Sinderen D, et al. Identification of universal gut microbial biomarkers of common human intestinal diseases by meta-analysis. FEMS Microbiol Ecol. 2017;93(12):fix153.

58. Beaumont M, Goodrich JK, Jackson MA, Yet I, Davenport ER, Vieira-Silva S, et al. Heritable components of the human fecal microbiome are associated with visceral fat. Genome Biol. 2016:17(1):189.

59. He J, He Y, Pan DD, Cao J, Sun YY, Zeng X. Associations of gut microbiota with heat stress-induced changes of growth, fat deposition, intestinal morphology and antioxidant capacity in ducks. Front Microbiol. 2019;10:903.

60. Leng Y, Yi M, Fan J, Bai Y, Ge Q, Yao G. Effects of acute intra-abdominal hypertension on multiple intestinal barrier functions in rats. Sci Rep. 2016; 6(1):22814.

61. Kisuse J, La-ongkham O, Nakphaichit M, Therdtatha P, Momoda R, Tanaka M, et al. Urban diets linked to gut microbiome and metabolome alterations in children: a comparative cross-sectional study in Thailand. Front Microbiol. 2018;9:1345.

62. Atarashi K, Tanoue T, Oshima K, Suda W, Nagano Y, Nishikawa H, et al. T reg induction by a rationally selected mixture of clostridia strains from the human microbiota. Nature. 2013;500(7461):232.

63. Satokari R, Fuentes S, Mattila E, Jalanka J, De Vos WM, Arkkila P. Fecal transplantation treatment of antibiotic-induced, noninfectious colitis and long-term microbiota follow-up. Case Rep Med. 2014;2014.

64. Grazul H, Kanda LL, Gondek D. Impact of probiotic supplements on microbiome diversity following antibiotic treatment of mice. Gut Microbes. 2016;7(2):101-14

\section{Ready to submit your research? Choose BMC and benefit from:}

- fast, convenient online submission

- thorough peer review by experienced researchers in your field

- rapid publication on acceptance

- support for research data, including large and complex data types

- gold Open Access which fosters wider collaboration and increased citations

- maximum visibility for your research: over $100 \mathrm{M}$ website views per year

At $\mathrm{BMC}$, research is always in progress.

Learn more biomedcentral.com/submissions 